\title{
EPILEPSIA DIFICULDADE DE APRENDIZAGEM ESCOLAR
}

\author{
JORGIANE DO SOCORRO SOUZA SANTOS ${ }^{1}$
}

\begin{abstract}
Resumo:
Este artigo anseia demonstrar como a trajetória da epilepsia é inseparável da trajetória histórica e balizada por conceitos educacionais relevantes, tanto no que se refere ao diagnóstico quanto ao tratamento.A epilepsia é uma doença neurológica, com alteração temporária e regressar do funcionamento cerebral envolvendo parte ou os dois hemisférios cerebrais.. Além destas características, a epilepsia pode causar problemas no desenvolvimento cognitivo, revelando, assim, problemas de comportamento e aprendizagem, além das dificuldades psicossociais associadas ao estigma afetando o seu desenvolvimento como um todo e a qualidade de vida social e familiar..O artigo busca demonstrar que a falta de esclarecimento da população em relação à epilepsia causa atitudes discriminatórias a seu portador, comprometendo a melhoria deste, sua inclusão social, e, consequentemente, sua qualidade de vida. Com embasamento teórico se dará através de livros e artigos científicos visando à boa qualidade bibliográfica, no contexto educativo Reconduzindo-a ao prazer das novas aprendizagens e possibilidades
\end{abstract}

Palavras-chave: Epilepsia; Aprendizagem; Cognição;

\begin{abstract}
Abstrat
This article aims to demonstrate how the epilepsy trajectory is inseparable from the historical trajectory and is marked by relevant educational concepts, both regarding diagnosis and treatment. Epilepsy is a neurological disease, with a temporary alteration and a return of cerebral functioning involving Part or both cerebral hemispheres. In addition to these characteristics, epilepsy can cause problems in cognitive development, thus revealing behavioral and learning problems, besides the psychosocial difficulties associated with stigma, affecting its development as a whole and the quality of life Social and family life. The article seeks to demonstrate that the lack of clarification of the population in relation to epilepsy causes discriminatory attitudes to its bearer, compromising its improvement, its social inclusion, and, consequently, its quality of life. With theoretical foundation will be given through books and scientific articles aiming at the good bibliographical quality, in the educational context Returning it to the pleasure of the new learnings and possibilities
\end{abstract}

Keywords: Epilepsy; Learning; Cognition;

\footnotetext{
${ }^{1}$ Licenciada em Pedagogia pela UNAMA-Universidade da Amazônia. Pós -Graduada em Educação Especial Inclusiva FPA e Psicopedagogia..E-mail:jorgianesss@hotmail.
} 


\section{INTRODUÇÃO}

O presente estudo está centrado na epilepsia na dificuldades acadêmicas dentro do contexto escolar. Paralelamente, algumas pesquisas mostram fatos de que as variáveis da epilepsia podem interferir no desempenho acadêmico, além de estarem significativamente relacionadas ao tipo de instituição de ensino (escola regular ou especial) que as crianças epilépticas frequentam. A epilepsia quase sempre se estende por muitos anos e acaba afetando, por um longo período, o desenvolvimento (Souza, 1999).Que por meio de uma atuação diferenciada e pautada na formação do cidadão de uma forma ampla. Neste processo de busca e reflexão faz-se necessário uma importante investigação: qual é impacto da doença e de suas variáveis no desenvolvimento acadêmico dessas crianças?

Além disso, vivemos a era da integração em todos os setores que se expressa de maneira mais ampla, pelo fenômeno da globalização da necessidade de ajuda, dos trabalhos realizados, das dificuldades detectadas, dos comportamentos e atitudes ou seja, auxilia no desenvolvimento de projetos favoráveis às mudanças educacionais, visando à descoberta e o desenvolvimento das capacidades da clientela, bem como poder contribuir para que os alunos sejam capazes de olhar esse mundo em que vive de saber interpretá-lo e de nele ter condições de interferir com segurança e competência.

Entendemos que é possível levar para dentro da sala de aula situações que favoreçam o melhor entendimento dos alunos que os jogos podem ser simples mais eficaz no contexto escolar, buscado instrumento de articulações, isto é estratégias de intervenção bem planejada.

\section{Epilepsia: concepção histórica}

Desde a Antiguidade até os dias atuais, no campo da Epileptologia, verificou-se um enorme progresso do conhecimento médico, com melhor compreensão dos mecanismos subjacentes à doença e maiores possibilidades de abordagem diagnóstica e terapêutica. No entanto, apesar desta grande evolução da Medicina, os epiléticos continuam a ser alvo de discriminação um 
pouco por todo o mundo, tanto por falta de informação, como por persistência de crenças antigas. A palavra epilepsia tem origem grega e significa simplesmente "ser pego" ou "ser atacado", fazendo referência à forma inesperada como se manifesta. A epilepsia possui registros escritos que datam de mais de 2000 anos antes de Cristo, caracterizando-se como o mais antigo distúrbio encefálico conhecido. As associações internacionais e nacionais, de que é um bom exemplo a Liga Portuguesa Contra a Epilepsia, desempenham um papel preponderante na informação e integração destes doentes na comunidade. Na História da Medicina Portuguesa foram encontradas inúmeras referências à epilepsia. Foi possível constatar a atenção cuidada que os profissionais nacionais dispensavam a esta doença e conhecer a sua experiência e as suas iniciativas, como promotores, da socialização dos epiléticos.

Por mais que a ciência progrida e se difunda, a imaginação, a criatividade e a sede de encontrar respostas pelo ser humano para o inexplicável conduzirão à persistência de crenças no paranormal. Por isso, a dicotomia natural/sobrenatural continuará a fazer parte de todos os tempos. Impunha-se relembrar a História da Epilepsia por constituir um exemplo do progresso científico e da humanização entre as doenças do foro neurológico. A Medicina, de mãos dadas com outras ciências, em particular as Ciências Sociais, vê culminar o esforço do seu trabalho com a afirmação dos recursos necessários à plena integração do doente epilético em sociedade.

Para Fernandes (2005), diferentes variáveis podem estar relacionadas ao surgimento destes problemas, como o tipo de epilepsia e sua severidade, a idade de início das crises, mas também a visão e as crenças da população em geral incluindo pais, professores, colegas de escola, que acreditam que as crianças epilépticas apresentam mais problemas de comportamento mesmo quando possuem repertório educacional e inteligência semelhantes aos de outras crianças saudáveis.

\section{EPILEPSIA DEFINIÇÃO}

Gareiso \& Escardó (1949, p.22) conceituaram a epilepsia da seguinte maneira: 


\begin{abstract}
"A epilepsia é um quadro clínico produzido por uma descarga elétrica súbita, anormal e desordenada dos neurônios. Essas descargas podem compreender uma, várias ou todas as categorias e níveis do sistema nervoso, assim fala de descargas psíquicas, descargas motoras, descargas sensitivas, descargas sensoriais e descargas neurovegetativas, todas as quais são expressão de epilepsia como conceito patogênico e constituem clinicamente as epilepsias."
\end{abstract}

Observa-se que as crianças com epilepsia podem se constituir como uns grupos educacionais vulnerável, uma vez diferentes variáveis podem estar relacionados ao surgimento de dificuldades escolares como o tipo de epilepsia e sua severidade, a idade de início das crises, a conotação psicossocial negativa da epilepsia, além da possibilidade de presença de estigma e reações inadequadas perante a epilepsia.

Segundo Dumas \& Giordano (1993, p.13):

\begin{abstract}
"Uma crise epiléptica é um fenômeno ágil; em geral breve e transitório. É uma crise cerebral que resulta numa descarga excessiva (como uma descarga elétrica) de um grupo mais ou menos vasto de células nervosas chamadas neurônios. Esse fenômeno é resultante de descargas síncrona, anormal e excessiva de uma população de neurônios do sistema nervoso central (SNC)."
\end{abstract}

Gastaut (1973) definiu epilepsia como:

"Uma desordem crônica do cérebro por várias etiologias, caracterizada por crises recorrentes devido à descarga de neurônios cerebrais [...]. Crises epilépticas isoladas ou ocasionais, ocorrendo em doenças agudas, não devem ser classificadas como epilepsia."

A epilepsia é uma enfermidade crônica constituindo-se como a condição neurológica mais comum na infância. Pode-se dizer que a epilepsia é uma das desordens que mais afeta o desenvolvimento cognitivo e sócio-afetivo típico da criança.

O diagnóstico da epilepsia não se baseia em exames complementares, mas sim na história clínica relatada pelo próprio paciente e por seu cuidador. É necessário que as informações sobre a crise sejam detalhadas, como a parte do corpo que foi afetada, o tempo de duração, o período do dia em que ocorreu a crise e quantas vezes por dia ocorreu.

Gram (1990, p.17) afirmou que:

"Uma síndrome epiléptica é definida como um distúrbio epiléptico caracterizado por um conjunto de sinais e sintomas que ocorrem simultaneamente. Essas características incluem tipo(s) de crise(s), etiologia, anatomia, fatores precipitantes, idade de início, severidade, cronicidade, possível ciclismo diurno e circadiano das crises e prognóstico."

É caracterizada por crises epilépticas repetidas e não é contagiosa. Assim, podemos Gran 1990 distinguir dois tipos fundamentais de crises 
epilépticas: as "generalizadas", envolvendo todo o cérebro, e as "parciais", em que a disfunção se limita a uma área cerebral.

As crises parciais podem subdividir-se em "simples" e "complexas". As "simples", nas quais não há alteração da consciência, consistem de convulsões de um membro ou parte do mesmo, ou de sensações de formigueiros percorrendo um membro e sensações abdominais. Nas "complexas", há alteração do estado de consciência, o doente apresenta-se confuso ou faz gestos automáticos de mastigação ou continua a desempenhar a tarefa que estava a executar automaticamente; outras vezes, esses "automatismos" consistem em esfregar as mãos, andar sem rumo, mexer sem nexo na roupa e manipular indevidamente os objetos.

Qualquer doença crônica na infância acarreta riscos ao desenvolvimento físico, psíquico e cognitivo da criança, aumentando a morbidade psicossocial (SOUZA, 2001; WAKAMOTO et al., 2000). Entretanto, a epilepsia, enquanto doença crônica parece afetar a criança mais do que outras enfermidades tais como a asma ou o diabetes (AUSTIN et al., 1994), apresentando repercussão no comportamento e no aprendizado de crianças e adolescentes epilépticos (COSTA; MAIA FILHO; GOMES, 2009).

À primeira vista, todos os pacientes parecem muito vulneráveis às críticas do ambiente, principalmente de seus familiares, e indefesos, possivelmente por falta de uma opinião própria mais elaborada, ou seja, de maior maturidade emocional. Outros, porém, quando conseguem acreditar um pouco mais em seus recursos físicos ou mentais e que, ao mesmo tempo, recebem apoio moral de seus familiares, conseguem um nível relativamente bom de adaptação social, diminuindo a importância que davam aos riscos e à sua imagem pública, além de reduzir a ansiedade, o que por si só pode diminuir a freqüência das crises. Isto vem apoiar a pressuposição da melhor e mais adequada sobrevivência daqueles com maior capacidade adaptativa.

\section{TRANSTORNOS MENTAIS ASSOCIADOS À EPILEPSIA}

Conforme foi exposto, as crianças com epilepsia apresentam maior vulnerabilidade para o desenvolvimento de problemas acadêmicos e a melhor 
compreensão destas questões pode levar a intervenções mais eficazes no futuro. Como toda desordem crônica, a epilepsia infantil é uma enfermidade complexa que traz repercussões importantes em diversos âmbitos da vida da criança. Dessa forma, é necessário uma intervenção do neuropsicopedagogo junto a equipe de profissionais para investigar e reconhecer quais variáveis estão envolvidas com as dificuldades apresentadas pelas crianças epilépticas e que podem, direta e/ou indiretamente, influenciar seu funcionamento psicossocial, em suas habilidades sociais e educacionais.

De acordo com Sturniolo e Galletti (1994), crianças com epilepsia constituem-se como um grupo educacional vulnerável apresentando risco elevado para desenvolver transtornos específicos do aprendizado e consequentemente comprometimentos no rendimento acadêmico, além de ajustamento psicossocial pobre, o que pode resultar em abandono da escola. Isso ocorre, provavelmente, porque a epilepsia, como citado acima, afeta diretamente o sistema nervoso e consequentemente pode trazer conflitos na percepção, no movimento, na consciência e em outras funções corticais, podendo comprometer a qualidade de vida daqueles que convivem com este transtorno.

O desconhecimento sobre a doença associado às crenças da população fundamentam o preconceito que muitas vezes surge na própria família e estende-se a outros grupos sociais dos quais as crianças e adolescentes participam, fazendo com que eles sofram com o estigma e sejam alijados do direito de participar ativamente da sociedade. Quando os pacientes recebem o diagnóstico de epilepsia, alguns podem reagir com período de tristeza antes da adaptação, mas, após elaborar esta situação, tendem a voltar ao humor normal. Porém, há aqueles que desenvolvem um quadro depressivo com características de morbidade, quer como sintoma, ou como uma síndrome depressiva, ou mesmo como entidade nosológica associada, cabendo ao clínico diferenciar entre uma depressão de características orgânicas ou uma depressão reativa psicogênica, além daquela sem características patológicas. Alguns autores mostra que na epilepsia infantil as dificuldades acadêmicas estão ligadas a fatores orgânicos relacionados diretamente a própria 
enfermidade como a doença neurológica de base e suas limitações físicas e cognitivas, a idade de início, a frequência de crises, tipo de síndrome epiléptica e etiologia, grau de controle e respectiva frequência das crises e o risco de acidentes, além dos efeitos colaterais das terapêuticas medicamentosas (SABBAGH et al., 2006; BERG et al., 2005; TIDMAN; SARAVANAN; GIBBS, 2003; BULTEAU et al., 2000; WILLIAM et al., 2001).

A depressão, então, pode ser concomitante ao quadro epiléptico, ou pode precedê-lo ou sucedê-lo. Há que se considerar, ainda, os episódios depressivos, que desaparecem em poucas horas.Também torna-se necessário a equipe de neuropsicopedagogo saber diagnosticar os diversos subtipos de quadro epiléptico, ou seja, o tipo de crise e o tipo de síndrome epiléptica, para avaliar a melhor conduta terapêutica.

Alguns autores apontam que as alterações cognitivas e sociais apresentadas pelas crianças com epilepsia não estão somente relacionadas com déficit intelectual, mas também com a possibilidade da presença de estigma relacionado à doença e às reações inadequadas dos pais, professores e amigos, que limitam experiências significativas para o desenvolvimento cognitivo-afetivo. Indivíduos com epilepsia podem ter distúrbios de aprendizagem. Entre as alterações que podem ter relação com a epilepsia, há as de linguagem, que manifestam-se em diferentes momentos, na ausência de crise, na aura, ou na crise convulsiva. Entre as alterações de linguagem que podem estar presentes na epilepsia cita-se a disfasia do desenvolvimento, afasia aguda com alteração transitória da função cognitiva, afasia epiléptica adquirida, deterioração da linguagem na infância, afasia transitória, disartria, gagueira, fala ininteligível, perseveração, dislexia, parafasias, dificuldades na compreensão de palavras e da escrita, alteração na linguagem escrita, prejuízo semântico e sintático.

É possível que o aluno com necessidades educacionais especiais, mais especificamente, o epilético precisa ter acesso a todos os instrumentos e estratégias que respondam às suas necessidades peculiares: professores que saibam identificá-la, equipe de profissionais que saiba como orientar 
professores e familiares, acompanhando-os no processo de aprendizagem de seus alunos e filhos.

\section{EPILEPSIA DIFICULDADES ACADÊMICAS}

$\mathrm{Na}$ epilepsia as dificuldades acadêmicas estão atreladas a fatores orgânicos pautados abertamente a própria enfermidade como a doença neurológica de base e suas limitações físicas e cognitivas Crianças com epilepsia demonstram riscos para dificuldades no desenvolvimento social e cognitivo. Zelnik et al. (2001) concordam que indivíduos com epilepsia são mais propensos a ter problemas de aprendizagem. Maior risco para uma variedade de problemas de comportamento e aprendizagem, a idade de início, a frequência de crises, tipo de síndrome epiléptica e etiologia, grau de controle e respectiva frequência das crises e o risco de acidentes, além dos efeitos colaterais das terapêuticas medicamentosas e cirúrgicas.

Porém, variáveis que podem estar envolvidas no processo de escolarização como baixa expectativa dos pais e professores quanto ao sucesso da criança, possibilidade de rejeição dos mestres e colegas de escola e alterações na auto-estima da criança, também são fatores importantes que podem promover um menor rendimento escolar da criança com epilepsia (GUERREIRO et al., 2000; MAIA FILHO, GOMES; FONTENELLE, 2004).

Em relação ao funcionamento acadêmico, investigações mais profundas sobre a questão ainda são necessárias e sugere-se a realização de pesquisas qualitativas destinadas a identificar aspectos ligados ao processo de escolarização de crianças com epilepsia as quais devem investigar dificuldades e linitações que interferem no aprendizado e que poderiam causar interferência no rendimento acadêmico.

"Toda criança pode aprender a ler e a escrever, mas não em qualquer situação. Mas está claro, também que não é em qualquer situação para todas as crianças. As condições para que ocorra aprendizagem vão variar de acordo com seu período de formação, pois todo processo de 
aprendizagem deve estar articulado com a história de cada indivíduo." (LIMA, 2002, p. 15).

Distúrbio de Aprendizagem Perturbação ou falha na aquisição e utilização de informações ou na habilidade para a solução de problemas. Grupo heterogêneo de transtornos que se manifesta por dificuldades significativas na aquisição e uso da escrita, fala, leitura, raciocínio ou habilidade matemática, distúrbio de Aprendizagem As crianças portadoras de distúrbio de aprendizagem não são incapazes de aprender, pois os distúrbios não são uma deficiência irreversível. O que é mais importante e identificar o sofrimento desta criança conversando coma a família com os pais vários estudos têm sido feitos na tentativa de relacionar tipos específicos de crises à chance aumentada de desenvolver transtornos depressivos, sendo os resultados muitas vezes contraditórios ou inconclusivos.

\section{CONCLUSÃO}

A epilepsia é uma enfermidade crônica constituindo-se como a condição neurológica mais comum na infância. Pode-se dizer que a epilepsia é uma das desordens que mais afeta o desenvolvimento cognitivo e sócio-afetivo típico da criança. Tais fatores podem interferir tanto no desempenho acadêmico quanto no tipo de instituição de ensino que crianças com epilepsia frequentam. Com base aos levantamentos bibliográficos percebeu-se que a epilepsia representa, portanto um grande desafio para as escolas que estão sendo chamadas para levar em conta a ampla diversidade das características e necessidades dos alunos adotando um modelo nele centrado e não no conteúdo, com ênfase na aprendizagem e não apenas no ensino. No Brasil, o estudo de Aguiar et al. (2007) tinha como objetivo avaliar o impacto das crises epilépticas na escola. Os pesquisadores mostraram que dentre as cinquenta crianças que participaram do estudo, $80 \%$ já tinham perdido pelo menos um dia na escola por causa das crises; $46 \%$ dos pais acreditavam que o filho deveria deixar a escola imediatamente após a ocorrência de uma crise; $60 \%$ das famílias permitiram que seus filhos faltassem à escola mesmo que não tivessem ocorrido crises no respectivo dia. 
Observa-se que as crianças com epilepsia podem se constituir como um grupo educacional vulnerável, uma vez diferentes variáveis podem estar relacionadas ao surgimento de dificuldades escolares como o tipo de epilepsia e sua severidade, a idade de início das crises, a conotação psicossocial negativa da epilepsia, além da possibilidade de presença de estigma e reações inadequadas perante a epilepsia infantil. Tais fatores podem interferir tanto no desempenho acadêmico quanto no tipo de instituição de ensino que crianças com epilepsia frequentam. A possibilidade de frequentar escolas regulares e a necessidade de utilização de serviços de educação especial por parte de crianças com epilepsia parece depender da gravidade do quadro que essas crianças apresentam. Cabe ressaltar que esta temática é ainda pouco explorada no Brasil e que pouco se sabe sobre os aspectos referentes ao acesso aos serviços de educação especial por parte de crianças com epilepsia.

Dessa forma, é necessário desenvolver maiores estudos para investigar e reconhecer quais variáveis estão envolvidas com as dificuldades apresentadas pelas crianças epilépticas e que podem, direta ou indiretamente, influenciar seu funcionamento psicossocial, em suas habilidades sociais e educacionais; além de implantar programas de conscientização sobre a epilepsia para a população, em especial, pais e professores; e de acompanhar as crianças com essa patologia em seu desenvolvimento escolar.Adicionalmente, faz-se necessário à identificação de recursos e estratégias que facilitem a inserção dos alunos com epilepsia com escolas regulares ou que desenvolvam recursos capazes de facilitar o aprendizado de crianças epilépticas que frequentam escolas especiais ou utilizam serviços de educação especial. 


\section{REFERÊNCIAS}

DUMAS, M. \& GIORDANO, C. L'epilepsie . Paris: Hermann, éditeurs des sciences et des arts, [s.d.].

GAREISO, A. \& ESCARDÓ, F. La epilepsia en el ninõ: nuevos conceptos, nuevas técnicas, nuevos tratamientos. Buenos Aires: El Ateneo Editorial, 1949. GASTAUT, H. Dictionary of epilepsy . Genebra: World Health Organization, 1973.

GRAM, Lennart. Crises e síndromes epilépticas. Londres: The Lancet, 1990.

GUERREIRO, C.A.; GUERREIRO, M.M.; CENDES, F.; LOPES-CENDES, I.; editores. Epilepsia. São Paulo: Lemos; 2000.

http://www.sbnpp.com.br/neuropsicopedagogo-institucional-e-clinico-artigocomentado-art-29-do-codigo-de-etica-tecnico-profissional/

LIMA, E. S. Desenvolvimento e aprendizagem na Escola: aspectos culturais, neurológicos e psicológicos. São Paulo: Sobradinho, 2002.

MAIA FILHO, H.S.; GOMES, M.M.; FONTENELLE, L.M. Epilepsia na infância e qualidade de vida. J Epilepsy Clin Neurophysiol. 2004;10:87-92.

SABBAGH, S. E. et al. Impact of epilepsy characteristics and behavioral problems on school placement in children. Epilepsy \& Behavior, v. 9, p.573578, 2006.

SOUZA E.A.Qualidade de vida na epilepsia infantil. Arq Neuro-Psiquiatr. 1999;57:33-9.

ZELNIK, N.; SA'ADI, L.; SILMAN-STOLAR, Z.; GOIKHMAN, I. Seizure control and educational outcome in childhood-onset epilepsy. J Child Neurol. 2001;16:820-4. 